\title{
The Comparative Analysis of Suture versus Linear Stapler Pharyngeal Closure in Total Laryngectomy: A Prospective Randomized Study
}

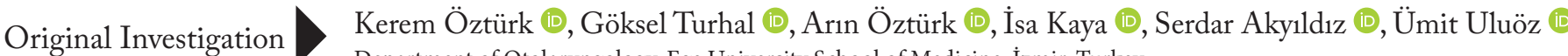 \\ Department of Otolaryngology, Ege University School of Medicine, İzmir, Turkey
}

\begin{abstract}
Objective: The aim of this study was to prospectively evaluate and compare the outcomes in patients undergoing pharyngeal closure with the linear stapler and suture techniques after total laryngectomy.

Methods: Forty-one patients who needed total laryngectomy for laryngeal cancer were enrolled in the study. Patients were randomized into two groups according to the pharyngeal closure technique: the linear stapler group (Group A) and the suture closure group (Group B). Rate of pharyngocutaneous fistula $(\mathrm{PCF})$, nasogastric tube (NGT) removal time and pharyngeal closure time were analyzed and compared between the two groups.

Results: PCF developed in three patients in Group A (14.3\%) and in seven patients in Group B (35\%). Although the rate of PCF was lower in Group A, this did not represent a statistical difference (p:0.129).
\end{abstract}

ORCID IDs of the authors:

K.0. 0000-0003-4608-6105;

G.T. 0000-0003-0020-1921;

A. $0.0000-0002-3321-7033$

i.K. 0000-0001-7096-4858;

S.A. $0000-0002-7472-5499$

Ü.U. 0000-0001-7498-5963.

Cite this article as: Öztürk K, Turhal G, Öztürk A, Kaya I, Akyıldıs S, Uluöz Ü. The Comparative Analysis of Suture versus Linear Stapler Pharyngeal Closure in Total Laryngectomy: A Prospective Randomized Study. Turk Arch Otorhinolaryngol 2019; 57(4): 166-70.

\section{Corresponding Author:}

Arın Öztürk, arinozturk@yahoo.com

Received Date: 28.05.2019

Accepted Date: 07.09.2019

Content of this journal is licensed under a Creative Commons Attribution 4.0 International License. Available online at www.turkarchotolaryngol.net

\section{Introduction}

Total laryngectomy is an ablative surgical technique applied in advanced-stage laryngeal cancer. Pharyngocutaneous fistula (PCF) is the most common early complication after total laryngectomy, with reported rates varying between $3 \%$ and $65 \%(1,2)$. In a meta-analysis that included 2598 patients from 16 studies, tumor subsite, T-stage, prior radiation, postoperative hemoglobin levels and status of the surgical margins were found as significant risk factors for PCF (3). In addition to the risk factors in the aforementioned study, Kiliç at al. (4) reported that age over 61 years, diabetes mellitus, alcohol use, preoperative tracheotomy, low levels of albumin, serum prealbumin levels on the third and seventh postoperative days, high postoperative C-Reactive Protein levels and primary surgery accompanied by bilateral neck dis-
Median NGT removal time was 12 days $(\mathrm{IQR}=3)$ and 19.5 days $(\mathrm{IQR}=1)$ in Groups $\mathrm{A}$ and $\mathrm{B}$, respectively. Median NGT removal time was not statistically different between the two groups ( $p: 0.642)$. All patients were able to swallow liquids and solids without difficulty. Median pharyngeal closure time was 3 minutes $(\mathrm{IQR}=1)$ and 37.5 minutes $(\mathrm{IQR}=9)$ in Groups $\mathrm{A}$ and $\mathrm{B}$, respectively. Median pharyngeal closure time was significantly lower in Group A (p:0.00).

Conclusion: The linear stapler technique in total laryngectomy is a reliable, safe, easy-to-apply and time-saving method. Closure with linear stapler significantly reduces pharyngeal closure time without adverse effect on NGT removal times and PCF rates.

Keywords: Total laryngectomy, pharyngocutaneous fistula, surgical stapler, larynx carcinoma section were also significantly associated for the development of postoperative PCF.

A meticulous closure technique to close the pharyngeal defect after total laryngectomy is important in preventing PCF. There are two main methods for neopharynx closure. While conventional manual suturing of the mucosal and the muscle layers is one, the other is linear stapler pharyngoplasty which is a novel technique and reduces surgery time to approximately 80 minutes and lowers the fistula rate (22.9\% vs $8.7 \%)$ (5).

The aim of the presented study was to prospectively evaluate and compare the outcomes of patients undergoing pharyngeal closure with the linear stapler or the suture technique after total laryngectomy. 


\section{Methods}

\section{Ethical Considerations}

All procedures involving human participants were performed in accordance with the ethical standards of the institutional and/or national research committee, and with the 1964 Helsinki Declaration and its more recent amendments or comparable ethical standards. The study was approved by the Ethics Committee of Ege University (IRB No. 15 - 12/6). Informed consent was obtained from all subjects.

\section{Study Design}

This study was carried out at the otolaryngology department of a tertiary medical center from August 2014 through June 2016. The study was designed as a prospective controlled study. All data were prospectively collected. These were demographic data of patients, date of surgery, type of surgery, type of pharyngeal closure, duration of the pharyngeal closure, complications and postoperative surgical follow-up.

\section{Patient Selection}

Forty-one patients who needed total laryngectomy for laryngeal cancer were enrolled in the study. Patients were randomized

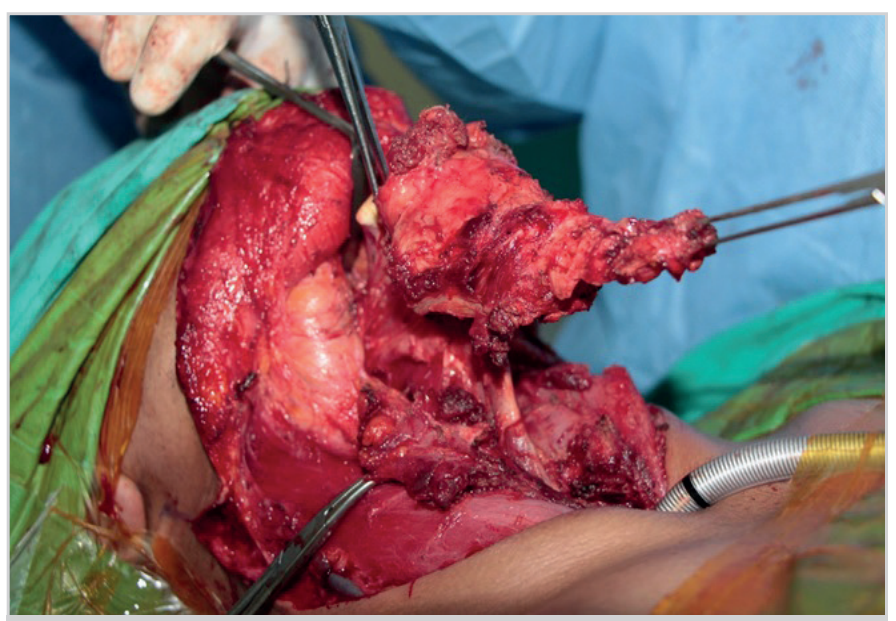

Figure 1. The larynx is grasped and elevated in the midline with two Allis clamps

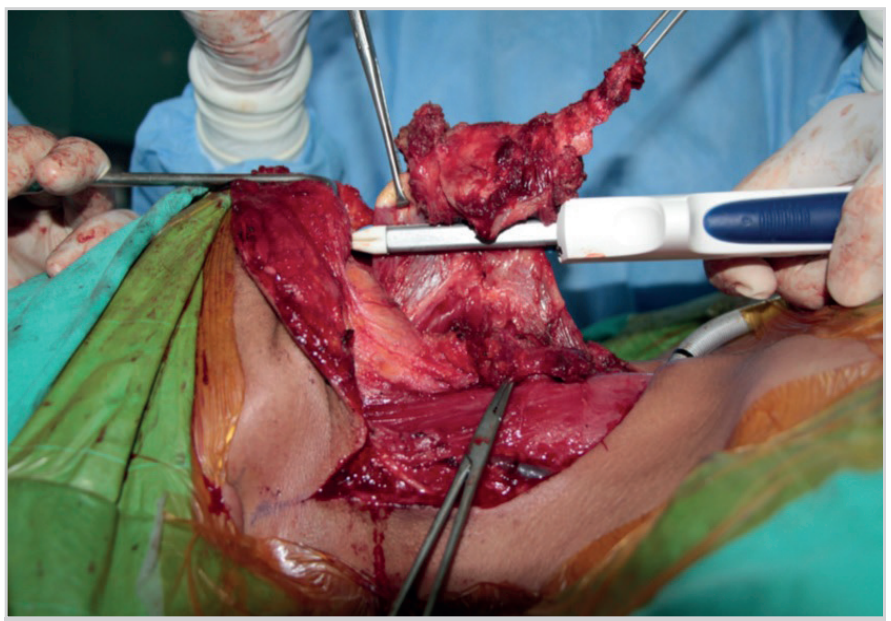

Figure 2. The linear stapler is placed over the pharyngeal mucosa into two groups according to the pharyngeal closure technique: The linear stapler group (Group A) and the suture closure group (Group B). There were 21 patients in Group A and 20 in Group B. All patients had biopsy-proven laryngeal cancer. Patients with vallecular, base of the tongue, post-cricoid and pyriform sinus extension were excluded in order to create two study groups with comparable characteristics. Additionally, to ensure homogeneous groups, patients with a history of radiotherapy and recurrent laryngeal cancer, and patients operated with bilateral, radical or modified radical type 1 or 2 neck dissections were excluded.

\section{Procedure}

All patients were operated on under endotracheal general anesthesia. All surgeries were performed by two staff surgeons experienced in the field of head and neck surgery. The surgeons also had experience with at least twenty cases in linear stapler closure. Prior to the surgeries the surgeons evaluated the $\mathrm{pa}^{-}$ tients' direct laryngoscopy reports, laryngoscopy videos and radiologic images to verify that closure could be performed with either of the techniques. Before the surgery, suspension laryngoscopy was performed to evaluate the extent of the tumor. Patients were consecutively randomized and assigned to one of the groups. After the larynx was removed and tumor-free surgical margins were obtained, the pharyngeal defect was closed with the linear $60 \mathrm{~mm}$ Covidien GIA6038S GIA ${ }^{\mathrm{TM}} 60-3.8$ Stapler (Medtronic; Minneapolis, USA) in Group A and with interrupted 3/0 vicryl sutures in Group B. Neck dissection was also performed as necessary. Linear stapler was applied after the tracheostomy was formed, the esophagus was freed from the laryngotracheal framework, and the larynx and pyriform sinuses were freed from the surrounding muscular and neurovascular tissues. Linear stapler was placed after the larynx was elevated by two Allis clamps. The epiglottis was grasped and retracted by an Allis clamp while the inferior part was elevated by retracting the anterior trachea with the other Allis clamp (Figure 1-3). The endolaryngeal view of the tumor after resection is shown in Figure 4. The larynx was then separated

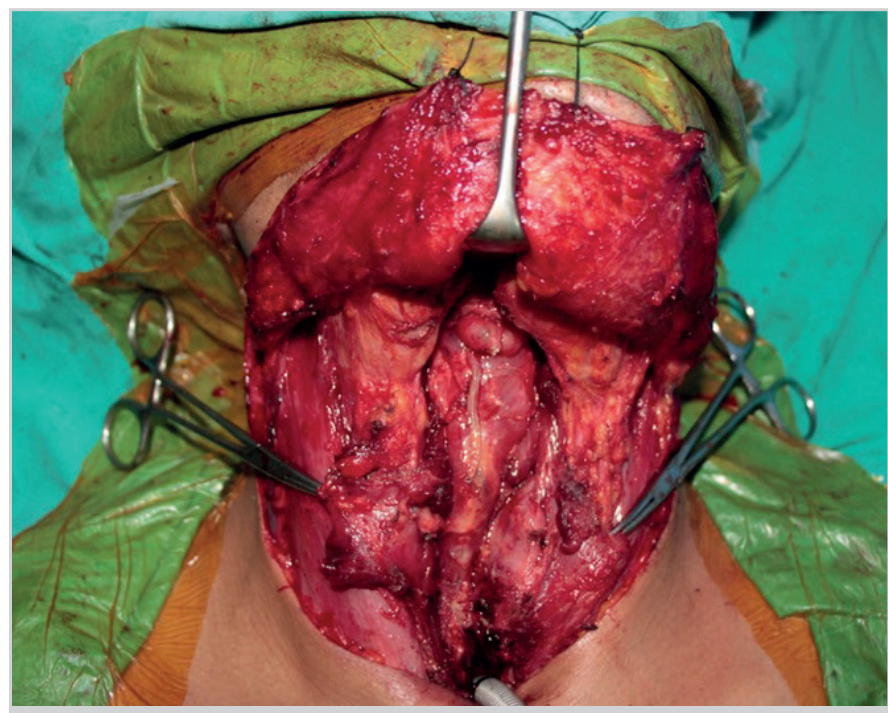

Figure 3. The suture line is shown 
from the pharyngeal mucosa by firing the linear stapler. One stapler cartridge was adequate in most of the patients, however, in patients with larger and longer larynges, two cartridges were needed. Pharyngeal closure time was calculated from the point when only the pharyngeal mucosa and esophagus remained attached to the larynx until the stapler closure or the last suture. Closure of the neck structures and the skin incisions was performed similarly in both groups. All patients were monitored closely for postoperative healing and complications. Primary tracheoesophageal puncture and voice prosthesis was not applied. We did not administer antibiotic prophylaxis, but intravenous ampicillin/sulbactam $8 \mathrm{~g} /$ day were administered to all patients until nasogastric tube (NGT) removal.

\section{Outcome Measures}

The rate of PCF, NGT removal time and pharyngeal closure time were analyzed and compared between the two groups.

Table 1. Demographic data of 41 patients

\begin{tabular}{l|c|c}
\hline & Age & Sex (Male:Female) \\
\hline Group A $(n=21)$ & $60.05 \pm 9.78 y$ & $20: 1$ \\
\hline Group B $(n=20)$ & $61.15 \pm 7.60 y$ & $18: 2$ \\
\hline Total $(n=41)$ & $60.59 \pm 8.69$ y (range 38-78) & $38: 3$ \\
\hline n: number, y: years &
\end{tabular}

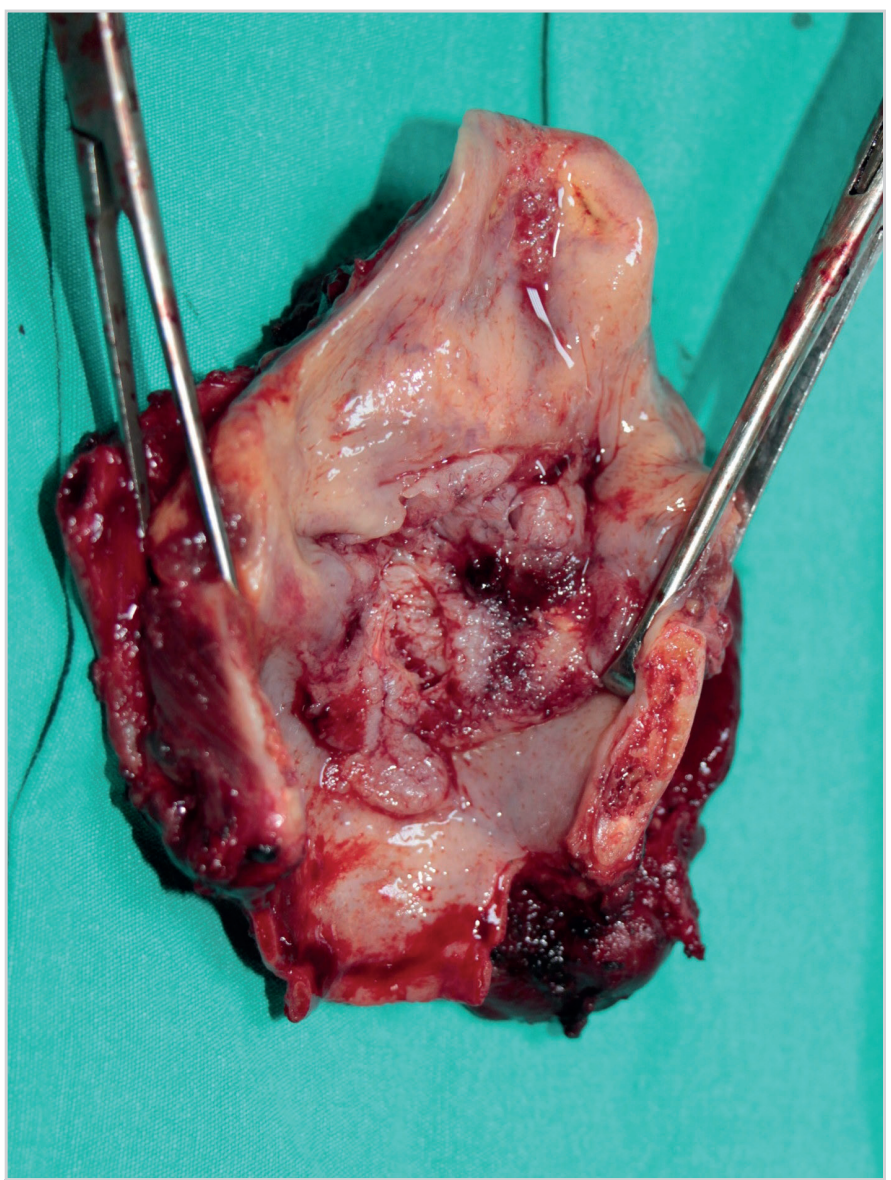

Figure 4. Total laryngectomy material. T3 tumor with anterior $(>1$ $\mathrm{cm})$ subglottic extension

\section{Statistical Analysis}

Statistical analysis was done using computer software SPSS version 22.0 (IBM Corp.; Armonk, NY, USA). Chi-square exact tests were used for the comparison of categorical data. Independent and paired sample t-tests were used for the analysis of parametric variables, while Wilcoxon and Mann-Whitney $\mathrm{U}$ tests were used for the analysis of non-parametric variables based on the distribution pattern of the data. The Shapiro-Wilk test was used for determining the distribution pattern of the data. The distribution of the groups was non-parametric. Correlation analysis was performed via Pearson's or Spearman's correlation analysis based on the distribution pattern of the data. Data were expressed as "median, interquartile range (IQR)". A $\mathrm{p}$-value of less than 0.05 was considered statistically significant.

\section{Results}

The study included 41 patients in total. The mean age of the 41 patients included in the study was $60.59 \pm 8.69$ (range 38-78 years, 38 males, 3 females). Patient ages did not significantly differ between the two groups (p:0.69). Demographic data are presented in Table 1. PCF developed in three patients in Group A (14.3\%) and in seven patients in Group B (35\%). Although the rate of $\mathrm{PCF}$ was lower in Group A, this did not represent a statistical difference (p:0.129). All patients who developed PCF were followed with conservative management with daily tight dressings. The median postoperative fistula closure period was 17 days $(\mathrm{IQR}=5)$ and 26 days $(\mathrm{IQR}=10)$ in Groups $\mathrm{A}$ and $\mathrm{B}$, respectively. Median postoperative PCF closure period was not statistically different between the two groups (p:0.735). Only one patient in group B underwent a secondary operation. PCFs were successfully closed with supraclavicular island flap after three-week follow-up period.

In Group A, 12 selective and nine modified radical type 3 neck dissections were performed, and in Group B, 13 selective and seven modified radical neck dissections were performed. Surgical margins were pathologically negative in all laryngectomy specimens.

Median NGT removal time was 12 days $(\mathrm{IQR}=3$ ) and 19.5 days (IQR=1) in Groups A and B, respectively. Median NGT removal time was not statistically different between the two groups (p:0.642). All patients were able to swallow liquids and solids without difficulty. Median pharyngeal closure time was 3 minutes $(\mathrm{IQR}=1)$ and 37.5 minutes $(\mathrm{IQR}=9)$ in Groups $\mathrm{A}$ and $\mathrm{B}$, respectively. Median pharyngeal closure time was significantly lower in Group A (p:0.00).

\section{Discussion}

Total laryngectomy causes a large defect in the pharyngeal mucosa. Watertight closure is required to prevent PCF. This closure can be achieved with conventional manual suturing or using a linear stapler. There are some beneficial effects of linear stapler closure in wound healing. Preservation of the mucosa, decontamination of the surgical field from saliva, and absence of necrosis are the primary advantages. In practice, however, there is not a certain neopharynx closure method, and prospective clinical studies are required for investigating the superiority of mechanical suturing in head and neck surgeries. 
Open, semi-closed and closed stapler pharyngeal techniques have been described (6-8). In the open techniques, as described by Talmi et al., (6) mucosal edges are closed with the stapler after laryngectomy. In the closed technique, as described by Bedrin et al., (7) the stapler is applied between the larynx and the pharynx after the larynx is skeletonized and the trachea is cut. The semiclosed technique was described by Altissimi and Frenguelli (8) to avoid trapping the suprahyoid part of the epiglottis between the jaws of the stapler. This technique differs from the closed technique by doing a mini pharyngostomy in the vallecular mucosa to retract the suprahyoid part of the epiglottis. The closed technique was adopted in our study because it offers a simpler approach and preserves the wound from contamination with saliva. Additionally, the suprahyoid epiglottis was not trapped in any of the cases.

PCF is the most common and probably one of the most bothersome complications of total laryngectomy. The rate of this complication is reported as high as $65 \%$ in earlier reports. PCF causes a significant increase in morbidity, prolongs hospital stay, and necessitates further interventions (9). Bedrin et al. (7) published their 25-year experience of using the linear stapler, which also represents the largest series with 1415 patients. They concluded that using a linear stapler was simple with a rapid application, provided watertight closure with good hemostasis, prevented field contamination, had no increase in fistula rate, and had good speech and deglutition outcomes. Likewise, Zhang et al. (10) reported a PCF rate of 4.76\%. Calli et al. (11) and Gonçalves et al. (12) reported significantly lower fistula rates with mechanical stapler closure after total laryngectomy. In a more recent study, Ismi et al. (13) also reported significantly lower fistula and surgical site infection rates with linear stapler compared to manual suturing. Recent prospective studies that compared manual suturing and mechanical closure of the pharynx reported the superiority of manual suturing on fistula rates. Babu et al. (14) reported the rate of PCF following linear stapler closure as $26.7 \% .75 \%$ of the patients that developed PCF had prior radiation and they defined this method as efficient and safe. Dedivitis et al. (15) reported higher fistula rates that were not statistically significant with linear stapler closure. In their conclusion, the authors mentioned that linear stapler use did not increase fistula rates. Fistula rates in these studies were relatively higher compared to more recent studies. The higher fistula rates were attributed to the low sample size, in which even one patient with $\mathrm{PCF}$ can significantly influence the rate.

Preoperative evaluation is of utmost importance before proceeding to stapler closure because the tumor is not visualized during resection. If the patient is not suitable for this technique, there is a potential risk for positive or close surgical margins. Additionally, using frozen sections after resection for the evaluation of the surgical margins becomes difficult and unsuitable. We performed preoperative suspension laryngoscopy to all patients to evaluate the extent of the tumors. This technique is more suitable for endolaryngeal tumors. Tumors that involve the vallecula, the base of the tongue, the post cricoid region, and the pyriform sinus are clear contraindications for stapler closure. Additionally, involvement of the suprahyoid epiglottis and the aryepiglottic folds poses a risk for oncologic compromise (11).

Shortened operating time is another benefit of this technique. Calli et al. (11) reported significant shorter operating times in their mechanical suturing group $(98.70 \pm 31.16$ minutes vs $506.99 \pm 24.12$ minutes; $p<0.001$ ). Zhang et al. $(10)$ reported that stapler closure saved 45 minutes compared to manual suturing. In our study, the total time of surgery was not analyzed because of the differences in neck dissection type and anatomy among the patients. Closure time, however, was found significantly lower in the linear stapler group.

The low number of patients is a limitation of our study. In the era of organ preservation, fewer patients are treated with total laryngectomy. Moreover, a significant number of total laryngectomy patients are operated on for failures in organ preservation protocols. These patients had to be excluded from our study because of the patient selection criteria. All patients in both groups were able to swallow both liquid and solids without difficulty in the postoperative period. Unfortunately, a clinical test evaluating deglutition such as videofluoroscopy or a swallowing assessment questionnaire is lacking in this study. Additionally, assessment and comparison of deglutition between the two groups require a long follow-up and individual factors like postoperative radiotherapy. We excluded patients with radiation history to perform a more proper comparison with homogeneous groups. We observed that using the linear stapler during total laryngectomy is a reliable, safe, easy-to-apply and time-saving technique. In the postoperative period, we can assume that these potential benefits directly affect the outcome. Analysis of cost-efficiency due to the reduced comorbidities provided by these techniques was not the topic of the study, but a better healing period was observed in patients in Group A, the linear stapler closure group. Given the advantageous technical benefits, this technique should be in the armamentarium of head and neck surgeons.

\section{Conclusion}

Closure of pharyngeal defect following total laryngectomy with the linear stapler technique significantly reduces pharyngeal closure time without adverse effects on NGT removal time and PCF rates.

Ethics Committee Approval: Ethics committee approval was received for this study from the Ethics Committee of Ege University (IRB No. $15-12 / 6)$.

Informed Consent: Written informed consent was obtained from the patients who participated in this study.

Peer-review: Externally peer-reviewed.

Author Contributions: Concept - K.Ö., G.T., İ.K.; Design - K.Ö., G.T., I.K., S.A; Supervision - K.Ö., Ü.U, S.A.; Fundings - Ü.U., S.A.; Materials - K.Ö, G.T, A.Ö.; Data Collection and/or Processing - K.Ö, G.T, A.Ö.; Analysis and/or Interpretation - K.Ö., G.T, A.Ö.; Writing - K.Ö, G.T, A.Ö.; Critical Reviews - K.Ö., Ü.U, S.A, İ.K. 
Öztürk et al.

Conflict of Interest: The authors have no conflicts of interest to declare.

Financial Disclosure: The authors declared that this study has received no financial support.

\section{References}

1. Bresson K, Rasmussen H, Rasmussen PA. Pharyngo-cutaneous fistulae in totally laryngectomized patients. J Laryngol Otol 1974; 88: 835-42. [CrossRef]

2. Papazoglou G, Doundoulakis G, Terzakis G, Dokianakis G. Pharyngocutaneous fistula after total laryngectomy: incidence, cause, and treatment. Ann Otol Rhinol Laryngol 1994; 103: 8015. [CrossRef]

3. Liang JW, Li ZD, Li SC, Fang FQ, Zhao YJ, Li YG. Pharyngocutaneous fistula after total laryngectomy: a systematic review and meta-analysis of risk factors. Auris Nasus Larynx 2015; 42: 353-9. [CrossRef]

4. Kiliç C, Tuncel U, Cömert E. Pharyngocutaneous fistulae after total laryngectomy: analysis of the risk factors and treatment approaches. B-ENT 2015; 11: 95-100.

5. Aires FT, Dedivitis RA, Castro MA, Bernardo WM, Cernea CR, Brandão LG. Efficacy of stapler pharyngeal closure after total laryngectomy: a systematic review. Head Neck 2014; 36: 739-42. [CrossRef]

6. Talmi YP, Finkelstein Y, Gal R, Shvilli Y, Sadov R, Zohar Y. Use of a linear stapler for postlaryngectomy pharyngeal repair: a preliminary report. Laryngoscope 1990; 100: 552-5. [CrossRef]
7. Bedrin L, Ginsburg G, Horowitz Z, Talmi YP. 25-year experience of using a linear stapler in laryngectomy. Head Neck 2005; 27 : 1073-9. [CrossRef]

8. Altissimi G, Frenguelli A. Linear stapler closure of the pharynx during total laryngectomy: a 15-year experience (from closed technique to semi-closed technique). Acta Otorhinolaryngol Ital 2007; 27: 118-22.

9. Westmore GA, Knowles JE. The use of a stapling instrument for postlaryngectomy pharyngeal repair. J Laryngol Otol 1983; 97: 775-8. [CrossRef]

10. Zhang X, Liu Z, Li Q, Liu X, Li H, Liu W, et al. Using a linear stapler for pharyngeal closure in total laryngectomy. Eur Arch Otorhinolaryngol 2013; 270: 1467-71. [CrossRef]

11. Calli C, Pinar E, Oncel S. Pharyngocutaneous fistula after total laryngectomy: less common with mechanical stapler closure. Ann Otol Rhinol Laryngol 2011; 120: 339-44. [CrossRef]

12. Gonçalves AJ, de Souza JA Jr, Menezes MB, Kavabata NK, Suehara $\mathrm{AB}$, Lehn $\mathrm{CN}$. Pharyngocutaneous fistulae following total laryngectomy comparison between manual and mechanical sutures. Eur Arch Otorhinolaryngol 2009; 266: 1793-8. [CrossRef]

13. Ismi O, Unal M, Vayisoglu Y, Yesilova M, Helvaci I, Gorur K, et al. Stapler esophageal closure during total laryngectomy. J Craniofac Surg 2017; 28: e35-e40. [CrossRef]

14. Babu S, Varghese B T, Iype E M, George P S, Sebastian P. Evaluation of stapled closure following laryngectomy for carcinoma larynx in an Indian tertiary cancer centre. Indian J Cancer 2015; 52: 37680. [CrossRef]

15. Dedivitis RA, Aires FT, Pfuetzenreiter EG Jr, Castro MAF, Guimarães AV. Stapler suture of the pharynx after total laryngectomy. Acta Otorhinolaryngol Ital 2014; 34: 94-8. 